\title{
Research on Formation Factors of Interdisciplinary Collaborative Innovation Network in Universities Based on Exponential Random Graph Model
}

\author{
Wang Limei \\ Beijing University of Technology, School of Economics and Management \\ Beijing 100124
}

\begin{abstract}
Based on three national awards data from 2011 to 2016, interdisciplinary collaborative innovation network in universities is constructed and the structure effects and exogenous effects that influence the formation of innovation network are put forward. An exponential random graph model (ERGM) is set up to identify factors that influence the formation of interdisciplinary collaborative innovation network in universities by model optimization. The results show that cooperation centrality, regional homogeneity and connectivity play positive roles in the formation of innovation network. Cooperation openness and experience have no significant influence and geographic proximity shows convergence trends. It provides a decisive reference for the development of interdisciplinary cooperation among universities.
\end{abstract}

Keywords-Collaborative innovation network; Exponential random graph model; Formation factors; Interdisciplinary

\section{INTRODUCTION}

The interdisciplinary development among universities is the collaborative innovation for various disciplinarians inside universities as well as the universities based on the same discipline or different disciplines [1], and the essence is constituted by the scientific research personnel of different disciplines, with the scientific research project being taken as a carrier and the solution of certain specific research task as an objective, and the interdisciplinary, pioneering and applicability thereof can meet the requirements of social development [2], and it is the part with the most innovation strength and vitality in innovative network. The interdisciplinary collaborative innovation among universities possesses complicated and dynamic characteristics, and can form network form; such network possesses competition and cooperation, collaborative integration, dynamics and openness [3], complexity, complementarity and other characteristics [4]. Therefore, it is of great importance to study the driving factors for the interdisciplinary collaborative innovation network of universities considering the sound and stable development of network.

\section{REFERENCE REVIEW}

The existing research about the driving factors of network formation is mainly carried out from network structure, organization individual attribute and inter-organizational relationship proximity [5], of which it can be found out through studying the driving factors of network formation from the perspective of network structure: the assortativity [6] of node and the network route length [7], the network scale, network connection strength and network centrality[1], the absorption ability of network structure [9-10], etc. can exert important influences on the formation and development of interdisciplinary collaborative innovation network among universities. Through studying the driving factors for the network formation based on node attribute, it can be obtained that: knowledge skills, professional experience [11], capacity "heterogeneity" [12-13], leading style [14], etc. are one of the main motivations for collaborative innovation. Besides, the relationship [15] between regional innovation capacity and the interdisciplinary collaborative cooperation network diffusion strength also has an important influence on network formation.

Over the recent years, the development of exponential random graph has gained wide attentions in the explorative research about the formation and factors of complicated network. The exponential random graph is put forward by Pattison and Wasserman [16], and this model has comprehensively considered the network endogenous structure and the relations of actors in the network, and can be used to flexibly analyze the network formed in the decision-making process of participators. In 2016, An W [17] put forward that the application of exponential random graph model in large-scaled social network analysis can essentially change the research direction of quantitative analysis, and transferred the research emphasis from individuals to network relationship and interaction.

This article takes the interdisciplinary collaborative innovation network among universities as the research object, puts forward the relevant assumption about the driving factors for network formation, integrates node attribute, network structure and concomitant variables, constructs exponential random graph model, analyzes the driving factors for forming the dynamic network of interdisciplinary collaborative innovation; meanwhile, it takes the innovation network formed for the national three big awards data in 2016 as an example for demonstration. 


\section{MODEL THINKING AND CONSTRUCTION}

Exponential random graph model (ERGM) is a kind of statistical method relying on network data fitting actual network structure [18]. ERGM uses the general exponential form [19] similar to logistic regression, comprehensively considers the node attribute in social influence model and the network topology in social selection model, applies Monte Carlo Markov's maximum likelihood estimation (MCMC-MLE) method to evaluate the variable parameters, and then through constant iterative process, it can construct random graph probability based on node attribute and network topology structure, and by virtue of Akaike Information Criterion (AIC) and Bayesian Information Criterion (BIC), select the most simple model, obtain the best association of variable, and the smaller these two values are, the closer the artificial network is from the observation network [20]. Finally, through comparing the statistical average of artificial network and the statistical value of observation network, conduct model evaluation [21].

The general form of exponential random graph model is:

$$
P_{\theta, y}(Y=y)=\frac{\exp \left\{\theta^{T} g(y)\right\}}{k(\theta, y)}
$$

Of which $Y$ is the artificial network generated by the model, $y$ is the actual network, $k(\theta, y)$ is the normalized constant term, $\theta$ is the model parameter assembly, and $g(y)$ is the statistical value of actual network. On the basis of general form, it is introduced into the analysis of driving factors for the interdisciplinary collaborative innovation network of universities. Assuming that the node of one network is $N=\{1,2,3, \cdots \cdots n\}, J=\{(i, j): i, j \in N, i \neq j\}$, which indicates all the possible relations of random network nodes. $E$ indicates the edge of the collaborative innovation network, and $E$ is a subset of $J$; random variable $Y$ indicates the element of $J$, and if $(i, j) \in E$ means that there is cooperation between subject $i$ and subject $j$ and $y_{i, j}=1$, otherwise $y_{i, j}=0$; in case of using $y=\left[y_{i, j}\right]$ to express the adjacent matrix of random network, all adjacent matrixes constitute the feasible set $Y$ of network adjacent matrix; $w_{i, j}$ indicates the cooperation numbers between subject $i$ and $j$, to constitute collaborative innovation network weight matrix $W_{i, j}=\left[w_{i, j}\right]$. Set $X$ as the condition assembly of random network, $x$ as the statistical value of actual network, and in consideration of the fact that the cooperation experience of the subject and the cooperation openness possess time-order characteristics in cooperation innovation network, select historical cooperation experience and historical cooperation openness as the exogenous variable $x^{\prime}$ of the year, so under the condition of $X=x$, the probability of $y$ for ERGM model in $Y$ fitting actual network is:

$$
\operatorname{Pr}\left(Y=y \mid X=x / x^{\prime}\right)=\frac{1}{\kappa(\theta, \beta)} \exp \left\{\beta^{T}\left[g(y, x)+g\left(y, x^{\prime}\right)\right]+\theta_{1} z_{1}(y)+\theta_{2} z_{2}(y)+\cdots \cdots+\theta_{\mathrm{m}} z_{m}(y)\right\}
$$

In order to measure the relative significance of each variable, set $\theta\left(\theta_{1}, \theta_{2}, \cdots \cdots, \theta_{m}\right)$ as the estimated parameter of endogenous variables, $m$ is the quantity of endogenous variables, and $z_{1}(y), z_{2}(y) \cdots \cdots \cdot z_{m}(y)$ as the structural statistical value of each endogenous variable; $\beta$ is the assembly of estimated parameters for exogenous variables,
$\beta^{T}=\left[\beta_{1}, \beta_{2}, \cdots \cdots, \beta_{q}\right]^{T}, q$ is the quantity of exogenous variables; in order to ensure that the sum of probability for all random graphs in the parameter optimization process of probability mass function $\operatorname{Pr}\left(Y=y \mid X=x / x^{\prime}\right)$ is equivalent to 1 , set normalization constant as $\kappa(\theta, \beta)[16]$, and the formula is:

$$
\kappa(\theta, \beta)=\sum_{y \in \bigcup} \exp \left\{\beta^{T}\left[g(y, x), g\left(y, x^{\prime}\right)\right]+\theta_{1} z_{1}(y)+\theta_{2} z_{2}(y)+\cdots \cdots+\theta_{m} z_{m}(y)\right\}
$$

Of which $U$ is the total set of random network for endogenous variables and exogenous variables under different estimated parameter combination, and $g(y, x)$ indicates the assembly of statistical value of exogenous variables:

$$
g(y, x)=\left[p_{1}(x), p_{2}(x), \cdots \cdots, p_{q-2}(x)\right]^{T}
$$

Of which $p_{1}(x), p_{2}(x), \cdots \cdots, p_{q-2}(x)$ indicates the statistical value of $q-2$ exogenous variables, and $g\left(y, x^{\prime}\right)$ indicates the assembly of statistical value for the historical cooperation experience and cooperation openness:

$$
g\left(y, x^{\prime}\right)=\left[p_{q-1}\left(x^{\prime}\right), p_{q}\left(x^{\prime}\right)\right]^{T}
$$

Of which $p_{q-1}\left(x^{\prime}\right)$ is the statistical value for the historical cooperation experience of each subject, and $p_{q}\left(x^{\prime}\right)$ is the statistical value for the historical cooperation openness of each subject.

Finally, through the conspicuousness of endogenous and exogenous statistical values in ERGM and the estimate of parameter, analyze the driving factors for the formation of interdisciplinary collaborative innovation network among universities, and adopt Statnet [22] software of R language to conduct model calculation and processing. 


\section{ASSUMPTION AND VARIABLE DESCRIPTION}

\section{A. Model Assumption}

On the basis of the existing research, this article digs out the driving factors for the formation of interdisciplinary collaborative innovation network among universities from network structure, node and relationship variable attribute, and meanwhile, provides the following assumptions:

H1: the interdisciplinary collaborative innovation network among universities possesses connectivity.

$\mathrm{H} 2$ : the interdisciplinary collaborative innovation network among universities possesses convergence.

H3: the interdisciplinary collaborative innovation network among universities possesses diffusivity.
H4: cooperation experience can exert a promotion effect on the formation of collaborative innovation network.

H5: cooperation openness can exert a promotion effect on the formation of collaborative innovation network.

H6: regional homogeneity has certain influence on the formation of collaborative innovation network.

H7: geographical proximity can influence the formation of collaborative innovation network.

\section{B. Model Variables}

Take the variable declarations for ERGM in endogenous structure effect and exogenous effect into consideration, which is as shown in Table 1.

TABLE I ERGM MODEL VARIABLES

\begin{tabular}{|c|c|c|c|c|}
\hline Name of Variables & $\begin{array}{l}\text { Meaning in } \\
\text { Chinese }\end{array}$ & $\begin{array}{l}\text { Schematic } \\
\text { diagram }\end{array}$ & Statistical Value & $\begin{array}{c}\text { Support } \\
\text { Assumptions }\end{array}$ \\
\hline Edges & 边 & $\mathrm{\longrightarrow} \longrightarrow$ & $\sum_{i, j} y_{i, j}$ & $\begin{array}{l}\text { Constant } \\
\text { term }\end{array}$ \\
\hline \multicolumn{5}{|l|}{ Structural effect } \\
\hline Two-path & 2-路径 & & $\sum_{i} \sum_{j, h, j \neq h} y_{j, i} y_{i, h}$ & H1 \\
\hline Gwidegree & 几何加权入度 & $\begin{array}{l}a \\
O> \\
0\end{array}$ & $\begin{array}{l}\text { Gwidegree equivalent to } \\
\text { Node } i\end{array}$ & $\mathrm{H} 2$ \\
\hline Gwodegree & 几何加权出度 & $\stackrel{O}{O} \rightarrow 0$ & $\begin{array}{l}\text { Gwidegree equivalent to } \\
\text { Node } i\end{array}$ & H3 \\
\hline \multicolumn{5}{|l|}{ Node effect } \\
\hline Co-experience $\left(\delta^{1}\right)$ & 合作经验 & & $\sum_{i, j} y_{i, j} \delta_{i, t}^{1}$ & $\mathrm{H} 4$ \\
\hline Co-openness $\left(\delta^{2}\right)$ & 合作开放度 & & $\sum_{i, j} y_{i, j} \delta_{i, t}^{2}$ & H5 \\
\hline \multicolumn{5}{|c|}{ Concomitant variable effect } \\
\hline $\begin{array}{c}\text { Regional } \\
\text { homogeneity } \\
(\mathrm{g} 1-\mathrm{net})\end{array}$ & 区域同质性 & & $\sum_{i, j} y_{i, j} g 1_{i j, t}$ & H6 \\
\hline $\begin{array}{c}\text { Geographical } \\
\text { proximity(g2-net) }\end{array}$ & 地理邻近性 & & $\sum_{i, j} y_{i, j} g 2_{i j}$ & H7 \\
\hline \multicolumn{5}{|c|}{$\begin{array}{ll}\text { On the basis of the national three big award data from } 2011 & \text { is the geometric weight out-degree, reflecting network } \\
\text { 2016, this article constructs the interdisciplinary } & \text { diffusivity. Specific to exogenous variables, cooperation } \\
\text { llaborative innovation network among universities in 2016, } & \begin{array}{l}\text { experience adopts each subject cooperation frequency from } \\
\text { d through pre-processing 2,748 subjects and } 602 \text { edges, in }\end{array} \\
\text { twork structure variables, 2-path reflects the basic } & \begin{array}{l}\text { can be measured on the basis of the quantity of main } \\
\text { nnectivity of network; Gwidegree refers to 几何加权入度 }\end{array} \\
\text { Chinese, and as per all the mutual relations pointing to } & \begin{array}{l}\text { Boolean value for processing, and when subjects are within the } \\
\text { same province, regional homogeneity is } 1 \text {, otherwise it is } 0 .\end{array} \\
\text { rtain node in the network, make statistics about the } \\
\text { ometric weight of such node, and as can be seen from the } \\
\text { erall structure of the network, Gwidegree reflects the }\end{array}$} \\
\hline
\end{tabular}




\section{MODEL FITTING AND OPTIMIZATION}

\section{A. Model Optimization And the Simplest Model Selection}

Select endogenous variables and exogenous variables of model, apply MCMC-MLE method to conduct model parameter estimation through several iterations, calculate the statistical values of artificial network and observation network, and then by virtue of the goodness of fit (Gof), evaluate model fitting effect. Different variable combinations have different final network fitting result, and in order to obtain the best and simplest model, i.e., obtain the best variable combination, to meet the best model fitting, compare Akaike Information Criterion (AIC) and Bayesian Information Criterion (BIC), to evaluate the fitting result of several ERGMs [23]. The result is as shown in Table 2 .

TABLE II ERGM FITTING OPTIMIZATION PROCESS

\begin{tabular}{|c|c|c|c|c|}
\hline Model Generation & Model 1 & Model 2 & Model 3 & Model 4 \\
\hline Edge & $\begin{array}{c}-5.60 \\
(0.07 * * *)\end{array}$ & $\begin{array}{c}-5.54 \\
(0.07 * * *)\end{array}$ & & $-5.14\left(0.006^{* * *}\right)$ \\
\hline \multicolumn{5}{|l|}{ Structural effect } \\
\hline 2-path & $\begin{array}{c}0.08 \\
\left(0.05^{* * *}\right)\end{array}$ & $\begin{array}{c}0.07 \\
(0.07 * * *)\end{array}$ & & $0.04(0.0002 * * *)$ \\
\hline Gwidegree & $0.31(0.16)$. & $\begin{array}{c}0.68 \\
(0.12 * * *)\end{array}$ & & $2.12(0.12 * * *)$ \\
\hline Gwidegree & $0.39\left(0.16^{*}\right)$ & $\begin{array}{c}0.77 \\
(0.12 * * *)\end{array}$ & & $1.55(0.11 * * *)$ \\
\hline \multicolumn{5}{|l|}{ Node effect } \\
\hline $\begin{array}{l}\text { Cooperation } \\
\text { experience }\end{array}$ & & $0.003(0.003)$ & $-0.02(0.0008 * * *)$ & 0.001 (0.0007.) \\
\hline Cooperation openness & & $\begin{array}{r}0.001 \\
(0.0009)\end{array}$ & $0.04(0.02 * * *)$ & $0.003(0.002)$ \\
\hline \multicolumn{5}{|c|}{ Concomitant variable effect } \\
\hline Regional homogeneity & & & $0.52(0.03 * * *)$ & $1.68(0.006 * * *)$ \\
\hline Geographic proximity & & & $\begin{array}{c}-0.003 \\
(0.00001 * * *)\end{array}$ & $\begin{array}{c}-0.0001 \\
(0.000009 * * *)\end{array}$ \\
\hline AIC & 36631 & 36174 & 63880 & 35287 \\
\hline $\mathrm{BIC}$ & 36674 & 36239 & 63923 & 35373 \\
\hline
\end{tabular}

Through comprehensively considering these three kinds of different variables, Model 1 merely considers the endogenous structure of network; Model 2 considers the network structure variables and node effect (which is also called as the receiver effect and sender effect); Model 3 merely considers exogenous variable (i.e., node effect and relation effect); Model 4 considers the network structure, node attribute and relation effect. Through Table 2, it can be found out that, AIC and BIC for Model 4 are the smallest, indicating that Model 4 is the simplest model, so the multivariable combination effect in Model 4 is the best. This article will carry out the analysis on the driving factors for the formation of interdisciplinary collaborative innovation network among universities on the basis of Model 4.

\section{B. Model Result And Assumption Support}

As can be seen from Table 2, the edge is a constant, and the general coefficient is a minus, and higher obviousness can reflect that the interdisciplinary collaborative innovation network among universities is sparse network. 2-path has stronger obviousness in Model 4, and positive effect can indicate that the interdisciplinary collaborative innovation network among universities possesses connectivity, and it can exert a positive promotion effect on network formation, so Assumption $\mathrm{H} 1$ is supported. In combination with the geometric weight out-degree result, stronger diffusivity in the network can make the network possess connectivity, and the relatively big coefficient can indicate that it can exert a key effect on network formation, and meanwhile, reflect that collaborative innovation network possesses good cooperation radiation capacity and stronger clustering, so Assumption H3 is supported. The obviousness of Gwidegree in Model 4 is positive, which indicates the stronger clustering of collaborative innovation network; meanwhile, it can also exert 
an important promotion effect on network formation. Along with the gradual promotion of collaborative innovation policy, the effect of degree has an important influence in the collaborative network development process, so Assumption $\mathrm{H} 2$ is supported.

The cooperation experience in Model 4 shows positive effect, but the obviousness is lower and the coefficient is smaller, indicating that the influence of cooperation experience on the formation of interdisciplinary collaborative innovation network among universities, so Assumption H4 cannot be supported.

In Model 4, it is not found that the historical cooperation openness has any obvious influence on the formation of interdisciplinary collaborative innovation network among universities, so Assumption H5 cannot be supported.

Regional homogeneity has higher obviousness in Model 4, with positive effect, which can indicate that the probability for the occurrence of interdisciplinary collaborative innovation among universities of the same province is bigger, so Assumption H6 is supported.

The obviousness of geological proximity in Model 4 is negative, which indicates that geological proximity shows divergence in the formation of interdisciplinary collaborative innovation network among universities, but the coefficient is smaller, so geological proximity cannot promote the forming factors for the interdisciplinary collaborative innovation network among universities, so Assumption H7 cannot be supported.

\section{CONCLUSION AND SUGGESTIONS}

Utilize the national three big award data to construct interdisciplinary collaborative innovation network among universities, and put forward the driving factors influencing network formation and the relevant assumptions. Dig out the influencing factors for the network formation on the basis of random graph model. It can be obtained that: firstly, the interdisciplinary collaborative innovation network among universities has connectivity and clustering, and can exert important promotion effect on network formation; secondly, the cooperation experience and cooperation openness for network subjects don't have obvious influence on network formation; thirdly, regional homogeneity is the key factor promoting the development of collaborative innovation network, and the geological proximity shows up divergence. Fourthly, the diffusivity for the cooperation of universities can also exert a positive effect on network formation.

To reinforce the interdisciplinary collaborative innovation and cooperation among universities, the following countermeasures and suggestions are put forward:

Firstly, promote the stable development of interdisciplinary collaborative innovation among universities; reinforce the heterogeneous level for interdisciplinary resources in universities, improve the flexibility of collaborative innovation, promote the collaborative innovation partnership, and form stable collaborative innovation cooperation mode.

Secondly, improve the interdisciplinary collaborative innovation capacity of universities, realize regional resource sharing mechanism, promote interdisciplinary collaborative innovation alliance development, and further expand the scale of interdisciplinary collaborative development.

Thirdly, improve the knowledge innovation diffusivity, realize multi-layer collaborative innovation cooperation system, further optimize cooperation partners, and realize the continuous development of interdisciplinary collaborative innovation among universities.

\section{REFERENCE}

[1] Huang Hongkun and Li Chunyan. Explosive Analysis on the Operation Mechanism Leading Collaborative Innovation in Universities from the Perspective of Innovation Subject [J]. Technology \& Business, 2014, 14: 342 .

[2] Wang Fenglei. Explosive Analysis on the Current Status of Interdisciplinary Research and Development in Our Country_Reflection Based on Constructing National Interdisciplinary System of Our Country [J]. Researches in Higher Education of Engineering, 2013, (1): 98 -102.

[3] Huang Shengjie and Zhang Yi. Explosive Analysis on the Organization Mode and Network Characteristics of Industry-study-research Cooperation in Our Country [J]. Researches in Higher Education of Engineering, 2002 (6): 30-33.

[4] Zhou Zhitai. Research on the Collaborative Innovation Network Based on the Perspective of Economics [D]. Jilin University, 2013.

[5] Ruan Pingnan, and Gu Chunliu. Analysis on the Microcosmic Effect Route of Technical Innovation Cooperation Network Knowledge Flow_- Taking the Biological Medicine Field of Our Country as an Example [J]. Scientific Progress and Countermeasures, 2017, 34 (17): 22-27.

[6] Chen Wei, Zhou Wen, and Lang Yifu et al. Research on the Industry-study-research Cooperation Innovation Network Structure and Risks-Taking Ocean Energy Industry as an Example [J]. Science and Scientific Technology Management, 2014 (9): 59-66.

[7] Lin Qiuyue, Wang Wenping and Wang Jiaoli. Simulation Analysis on the Structural Characteristics for the Innovation Network of Industrial Cluster-Based on March Utilization Mode-Explosive Innovation Analysis Framework [J]. Journal of Management, 2010, 7 (7): 1015-1020.

[8] Xie Xuemei, Fang Liangxiu, Zeng Saixing. Collaborative Innovation Network and Knowledge Transfer Performance: AFs QCA Approach [J]. Journal of Business Research, 2016, 69: 5210-5215.

[9] Nie Miao. Optional Research on the Industry-study-research Cooperation Mode Based on Absorption Power of Enterprises [D]. Shanghai Jiaotong University, 2011.

[10] Qin Wei, and Xu Fei. Research on the Evolution of Industry-study-research Cooperation Mode Based on Absorption Power: Taking Baosteel-Shanghai Jiaotong University as an Example [J]. Science and Technology Management Research, 2010, 30(1):19-21.

[11] You Li. Empirical Research on the Relationship between the Knowledge Heterogeneity of Interdisciplinary Team in Universities and the Innovation Performance [J]. Journal of National Academy of Education Administration, 2017 (3): 62-69.

[12] Fang Ruguo, and Li Xing. Analysis on the Multi-enterprise Dynamic Cooperation Innovation Games within Industrial Cluster [J]. Learning and Practice, 2011, 12: 35-43.

[13] Arrow K. Economic Welfare and the Allocation of Resources for Invention [J]. The Rate and Direction of Inventive Activity: Economic and Social Factors, 1962: 609-626.

[14] Huang Shufang. Research about the Relation between the Team Heterogeneity of Interdisciplinary Cooperation and the Original Innovation Performance of Universities [D]. Zhejiang University, 2016 . 
[15] Liu Fangfng. Research on the Cross-regional Industry-study-research Cooperation under the Perspective of Social Network [D]. University of Science and Technology of China, 2016.

[16] Dean Lusher, John Koskinen, Gary Robbins. Exponential Random Graph Model of Social Network [M]. Social Sciences Academic Press, 2016.

[17] An W. Fitting ERGMs on big networks[J]. Social Science Research, 2016, 59:107.

[18] Holland P W, Leinhardt S. An Exponential Family of Probability Distributions for Directed Graphs [J]. Journal of the American Statistical Association, 1981, 76 (373): 33-50.

[19] Hunter D R, Handcock M S, Butts C T, et al. ergm: A Package to Fit, Simulate and Diagnose Exponential-Family Models for Networks[J]. Journal of Statistical Software, 2008a, 24(3):nihpa54860.

[20] Scott T A. Analyzing Policy Networks Using Valued Exponential Random Graph Models: Do Government-Sponsored Collaborative Groups Enhance Organizational Networks? [J]. Policy Studies Journal, 2015, 44 (2): 215-244.

[21] Lusher, D., Koskinen, J., Robins, G., Lusher, D., Koskinen, J., \& Robins, G. (2013).Exponential Random Graph Models for Social Networks. Structural Analysis in the Social Sciences. New York, NY: Cambridge University Press.

[22] Hunter D R, Goodreau S M, Handcock M S. ergm.userterms: A Template Package for Extending statnet [J]. Journal of Statistical Software, 2013, 52 (2): i02.

[23] Silk M J, Fisher D N, Silk M J, et al. Understanding Animal Social Structure: Exponential Random Graph Models in Animal Behavior Research [J]. Animal Behavior, 2017, 132: 137-146. 